\title{
Numerical investigation of the performance of an SPR-based optical fiber sensor in an aqueous environment using finite-difference time domain
}

\begin{abstract}
We investigate a surface plasmon resonance (SPR)-based optical fiber sensor using 2-D finite-difference time domain (FDTD) simulations. The optical sensor is designed by polishing a single-mode optical fiber by symmetrically removing a portion of its cladding forming two sensing regions. We analyze the effects of two physical parameters of the sensor in an aqueous medium, i.e. the thickness of the metal layer and the amount of residual cladding using numerical simulations. The results show that a good transmission dip can be obtained by optimizing these parameters. Thus, the sensor structure can be deployed as an optical biosensor in aqueous environments.
\end{abstract}

Keyword: Finite-difference time domain; Optical fiber sensor; Refractive index; Surface plasmon resonance 